\title{
THE GHIRLANDA-GUERRA IDENTITIES
}

\author{
Pierluigi Contucci ${ }^{\dagger}$, Cristian Giardinà ${ }^{\ddagger}$ \\ $\dagger$ Dipartimento di Matematica \\ Università di Bologna, 40127 Bologna, Italy \\ e-mail: contucci@dm.unibo.it \\ $\ddagger$ EURANDOM \\ P.O. Box 513 - 5600 MB Eindhoven, The Netherland \\ e-mail: giardina@eurandom.tue.nl
}

\begin{abstract}
If the variance of a Gaussian spin-glass Hamiltonian grows like the volume the model fulfills the Ghirlanda-Guerra identities in terms of the normalized Hamiltonian covariance.
\end{abstract}




\section{Introduction}

In the last decade new ideas and technical methods have been developed in the attempt to build the spin glass theory on rigorous mathematical grounds. The most recent example is the interpolation strategy $[\mathrm{GT}]$ introduced to prove the existence of the thermodynamic limit in the Sherrington-Kirkpatrick [SK] model and its use in the proof of the Parisi free energy $[\mathrm{MPV}]$ of the same model [G1, T1] and in the identification of an extended variational principle [AiSS].

The first basic contribution in the field came with the work by Guerra [G2] on how to prove some correlation identities of the Sherrington-Kirkpatrick model that were only assumed within the ultrametric structure of the Parisi solution. Those identities were later generalized by Ghirlanda-Guerra $[\mathrm{GG}]$ and are playing an increasingly important role in the mathematical approach to the low temperature spin glass phase (see [B, T2] and references therein). The Ghirlanda-Guerra identities are consequence of a very basic principle of statistical mechanics i.e. the vanishing of the fluctuation of the energy per particle: at increasing volumes the energy per particle approaches a constant with respect to the equilibrium measure. Within the non-disordered classical cases all that simply implies the finiteness of the specific heat almost everywhere in the temperature (see nevertheless the implications in classical mean-field models [CGI]); however in the spin glass cases, where the equilibrium quenched state is a properly intertwined composition of the Boltzmann-Gibbs and the disorder measures, its consequences are way more subtle. The work [G2] led to the identification of the stochastic stability $[\mathrm{AC}]$, an invariance property of the quenched state under a class of suitable perturbations. Subsequently stochastic stability was developed and used clarify the relation between the equilibrium and the off-equilibrium properties in the spin glass phase [FMPP1, FMPP2]. Recently stochastic stability has been classified $[\mathrm{C}, \mathrm{BCK}]$ and placed on rigorous grounds in [CGi], where its relation with the mentioned identities is also discussed.

In this work we obtain a condition that guarantee the validity of the Ghirlanda-Guerra identities: our result states that they hold true whenever the variance of the Hamiltonian function grows like the volume. Such a condition is the same that ensure the existence 
(boundedness) of the thermodynamic limit $[\mathrm{CG}]$ and applies to every spin glass model studied so far: to the Edwards-Anderson model, to the finite dimensional cases with summable or non-summable interactions in the sense of Kanin and Sinai [KS], to the mean field cases like SK, p-spin, REM and GREM, up to the general spin glass model of subset interaction on which our general condition has been tailored. It is important to stress that the identities we prove hold in terms of the normalized Hamiltonian covariance which has a different spin expression in each model: for instance in the SK model it coincides with the square power of the overlap function, while for the EA model it is the link overlap $[\mathrm{C} 2, \mathrm{NS}]$. The strategy we use to achieve the result relies on very simple methods like the bound on martingale sums and classical inequalities.

The paper is organized in definitions (Sec. 2), results (Sec. 3), proofs (Sec. 4) and is concluded with some comments and perspectives (Sec. 5).

\section{Definitions}

We consider a disordered model of Ising configurations $\sigma_{n}= \pm 1, n \in \Lambda \subset \mathbb{Z}^{d}$ for some $d$ parallelepiped $\Lambda$ of volume $|\Lambda|$. We denote $\Sigma_{\Lambda}$ the set of all $\sigma=\left\{\sigma_{n}\right\}_{n \in \Lambda}$, and $\left|\Sigma_{\Lambda}\right|=2^{|\Lambda|}$. In the sequel the following definitions will be used.

\section{Hamiltonian.}

For every $\Lambda \subset \mathbb{Z}^{d}$ let $\left\{H_{\Lambda}(\sigma)\right\}_{\sigma \in \Sigma_{N}}$ be a family of $2^{|\Lambda|}$ translation invariant (in distribution) centered Gaussian random variables defined, in analogy with [RU], according to the very general representation

$$
H_{\Lambda}(\sigma)=-\sum_{X \subset \Lambda} J_{X} \sigma_{X}
$$

where

$$
\sigma_{X}=\prod_{i \in X} \sigma_{i}
$$

$\left(\sigma_{\emptyset}=0\right)$ and the $J$ 's are independent Gaussian variables with zero mean

$$
\operatorname{Av}\left(J_{X}\right)=0
$$


and (translation invariant) variance

$$
\operatorname{Av}\left(J_{X}^{2}\right)=\Delta_{X}^{2}
$$

2. Covariance matrix.

$$
\mathcal{C}_{\Lambda}(\sigma, \tau):=\operatorname{Av}\left(H_{\Lambda}(\sigma) H_{\Lambda}(\tau)\right)=\sum_{X \subset \Lambda} \Delta_{X}^{2} \sigma_{X} \tau_{X}
$$

By the Schwartz inequality

$$
\left|\mathcal{C}_{\Lambda}(\sigma, \tau)\right| \leq \sqrt{\mathcal{C}_{\Lambda}(\sigma, \sigma)} \sqrt{\mathcal{C}_{\Lambda}(\tau, \tau)}=\sum_{X \subset \Lambda} \Delta_{X}^{2}
$$

for all $\sigma$ and $\tau$.

\section{Thermodynamic Stability.}

The Hamiltonian (2.1) is thermodynamically stable if it exist a constant $\bar{c}<\infty$ such that

$$
\sup _{\Lambda \subset \mathbb{Z}^{d}} \frac{1}{|\Lambda|} \mathcal{C}_{\Lambda}(\sigma, \sigma)=\sup _{\Lambda \subset \mathbb{Z}^{d}} \frac{1}{|\Lambda|} \sum_{X \subset \Lambda} \Delta_{X}^{2} \leq \bar{c}<\infty
$$

Together with translation invariance a condition like the (2.7) is equivalent to

$$
\sum_{X \ni 0} \frac{\Delta_{X}^{2}}{|X|} \leq \bar{c}
$$

In fact

$$
\sum_{X \subset \Lambda} \Delta_{X}^{2}=\sum_{x \in \Lambda} \sum_{X \ni x} \frac{\Delta_{X}^{2}}{|X|}=|\Lambda| \sum_{X \ni 0} \frac{\Delta_{X}^{2}}{|X|} .
$$

Alternatively, summing over the equivalence classes $\widetilde{X}$ of the translation group, the (2.7) is equivalent to

$$
\sum_{\widetilde{X}} \Delta_{\widetilde{X}}^{2} \leq \bar{c}
$$

Thanks to the (2.6) a thermodynamically stable model fulfills the bound

$$
\mathcal{C}_{\Lambda}(\sigma, \tau) \leq \bar{c}|\Lambda|
$$

and has a order 1 normalized covariance

$$
c_{\Lambda}(\sigma, \tau):=\frac{1}{|\Lambda|} \mathcal{C}_{\Lambda}(\sigma, \tau)
$$


4. Random partition function.

$$
\mathcal{Z}(\beta):=\sum_{\sigma \in \Sigma_{\Lambda}} e^{-\beta H_{\Lambda}(\sigma)}
$$

5. Random free energy.

$$
-\beta \mathcal{F}(\beta):=\mathcal{A}(\beta):=\ln \mathcal{Z}(\beta) .
$$

6. Random internal energy.

$$
\mathcal{U}(\beta):=\frac{\sum_{\sigma \in \Sigma_{\Lambda}} H_{\Lambda}(\sigma) e^{-\beta H_{\Lambda}(\sigma)}}{\sum_{\sigma \in \Sigma_{\Lambda}} e^{-\beta H_{\Lambda}(\sigma)}} .
$$

7. Quenched free energy.

$$
-\beta F(\beta):=A(\beta):=\operatorname{Av}(\mathcal{A}(\beta)) .
$$

8. R-product random Gibbs-Boltzmann state.

$$
\Omega(-):=\sum_{\sigma^{(1)}, \ldots, \sigma^{(R)}}(-) \frac{e^{-\beta\left[H_{\Lambda}\left(\sigma^{(1)}\right)+\cdots+H_{\Lambda}\left(\sigma^{(R)}\right)\right]}}{[\mathcal{Z}(\beta)]^{R}} .
$$

9. Quenched equilibrium state.

$$
<->:=\operatorname{Av}(\Omega(-))
$$

10. Observables.

For any smooth bounded function $G\left(c_{\Lambda}\right)$ (without loss of generality we consider $|G| \leq 1$ and no assumption of permutation invariance on $G$ is made) of the covariance matrix entries we introduce the random (with respect to $<->$ ) $R \times R$ matrix of elements $\left\{q_{k, l}\right\}$ (called generalized overlap) by the formula

$$
<G(q)>:=\operatorname{Av}\left(\Omega\left(G\left(c_{\Lambda}\right)\right)\right)
$$

$$
\begin{aligned}
& \text { E.g.: } G\left(c_{\Lambda}\right)=c_{\Lambda}\left(\sigma^{(1)}, \sigma^{(2)}\right) c_{\Lambda}\left(\sigma^{(2)}, \sigma^{(3)}\right) \\
& \qquad q_{1,2} q_{2,3}>=\operatorname{Av}\left(\frac{\sum_{\sigma^{(1)}, \sigma^{(2)}, \sigma^{(3)}} c_{\Lambda}\left(\sigma^{(1)}, \sigma^{(2)}\right) c_{\Lambda}\left(\sigma^{(2)}, \sigma^{(3)}\right) e^{-\beta\left[\sum_{i=1}^{3} H_{\Lambda}\left(\sigma^{(i)}\right)\right]}}{[\mathcal{Z}(\beta)]^{3}}\right)
\end{aligned}
$$




\section{Results}

The Ghirlanda-Guerra identities admit several equivalent formulations. They can be expressed in terms of factorization properties of the quenched distribution of the generalized overlap [GG, B, T2] as well as in terms of expectations of observables. In this work we chose the second approach because it allows to distinguish the identities in two classes with different physical meaning: the first expresses the regularity with respect to the temperature, the second the self-averaging of intensive quantities.

In relation to the definitions of the previous section it holds the following:

Theorem 1 The quenched equilibrium state of a thermodynamically stable Hamiltonian fulfills, for every observable $G$ and every temperature interval $\left[\beta_{1}^{2}, \beta_{2}^{2}\right]$ the following identities in the thermodynamic limit

$$
\begin{gathered}
\int_{\beta_{1}^{2}}^{\beta_{2}^{2}}<\sum_{\substack{k, l=1 \\
k \neq l}}^{R} G q_{l, k}-2 R G \sum_{l=1}^{R} q_{l, R+1}+R(R+1) G q_{R+1, R+2}>d \beta^{2}=0 \\
\int_{\beta_{1}^{2}}^{\beta_{2}^{2}}\left[\sum_{k=1}^{R}<G q_{k, R+1}>-(R+1)<G q_{R+1, R+2}>+<G><q_{1,2}>\right] d \beta^{2}=0
\end{gathered}
$$

Remark 1 The two previous relations when applied to $G(q)=q_{1,2}$ combined together lead to the well known [MPV, G2]:

$$
\begin{aligned}
& <q_{1,2} q_{2,3}>=\frac{1}{2}<q^{2}>+\frac{1}{2}<q>^{2} \\
& <q_{1,2} q_{3,4}>=\frac{1}{3}<q^{2}>+\frac{2}{3}<q>^{2}
\end{aligned}
$$

Remark 2 It is straightforward to verify that the condition (2.7) of thermodynamic stability holds for all the known spin glass models. Here are a few examples:

1. The Edwards-Anderson model [EA]. The nearest neighbor case is defined by $\Delta_{X}^{2}=1$ if $X=\left(n, n^{\prime}\right)$ and $\left|n-n^{\prime}\right|=1$. The condition (2.7) is verified by $\bar{c}=d$. 
2. More generally one consider still a two body interaction with $\Delta_{X}^{2}=\left|n-n^{\prime}\right|^{-2 d \alpha}$. The regime $\alpha>1$ comes from a summable interaction. The condition (2.7) is verified by $\bar{c}=(2 \alpha-1)^{-d}$ for all $\alpha>1 / 2$ thus including also the non summable case [KS].

3. The SK model [SK]. Although it is not a finite dimensional model it may still be embedded in $\mathbb{Z}$, with $\Delta_{X}^{2}=0$ unless $|X|=2$ and $\Delta_{i, j}=N^{-1}$ with $N=|\Lambda|$. It obviously fulfills condition (2.7) with $\bar{c}=1$.

4. The p-spin. Analogously as above $\Delta_{X}=0$ if $|X| \neq p$ and $\Delta_{X}^{2}=1 / N^{p}$ otherwise. It is thermodynamically stable with $\bar{c}=1$

5. The REM $[D]$ and GREM [DG] models. Although they have not been defined as spin models their discrete nature allows to associate to them a spin Hamiltonian. For instance it is easy to prove that the REM is represented by by the Hamiltonian (2.1) with $\Delta_{X}^{2}=N 2^{-N}$ which satisfies the condition (2.7) with $\bar{c}=1$, see also [B]. The same argument holds for the GREM [CDGG, CG2] which is again thermodynamically stable with $\bar{c}=1$.

Remark 3 The relevance of the identities is evident considering that they reduce the degrees of freedom a priori carried by each spin glass model. In the mean field case for instance the method led to the rigorous proof of a property called replica equivalence $[P a, C 2]$ which can viewed as an ansatz generalizing the ultrametric one. The purely ultrametric identities (still lacking a rigorous mathematical derivation) which are built in the Parisi solution of the SK model are not contained in the Ghirlanda-Guerra ones.

Remark 4 It would be interesting to establish, or disprove, the same identities in a stronger sense, i.e. everywhere in the temperature. One of the limit of the method used to achieve our results is that it is intrinsically restricted to hold in $\beta$-average, i.e. in every interval excluding at most isolated singularities. It is still an open question if, in the spin glass phase, there are similar singularities or if the identities hold just everywhere. The only existing results are evidences of numerical nature of the validity of those identities everywhere [MPRRZ, CGi2]. 


\section{Proof}

The statements (3.21) and (3.22) are proved respectively in the lemmata of subsections 4.1 and 4.2. The proof uses only elementary methods like martingale differences and classical inequalities. Let $h(\sigma)=|\Lambda|^{-1} H_{\Lambda}(\sigma)$ be the Hamiltonian per particle. We consider the quantity

$$
\sum_{l=1}^{R}\left\{<h\left(\sigma^{(l)}\right) G>-<h\left(\sigma^{(l)}\right)><G>\right\}=\Delta_{1} G+\Delta_{2} G
$$

where

$$
\begin{gathered}
\left.\Delta_{1} G=\sum_{l=1}^{R}\left\{\operatorname{Av}\left(\Omega\left[h\left(\sigma^{(l)}\right) G\right]-\Omega\left[h\left(\sigma^{(l)}\right)\right] \Omega[G]\right)\right)\right\} \\
\Delta_{2} G=\sum_{l=1}^{R}\left\{\operatorname{Av}\left(\Omega\left[h\left(\sigma^{(l)}\right)\right] \Omega[G]\right)-\operatorname{Av}\left(\Omega\left[h\left(\sigma^{(l)}\right)\right]\right) \operatorname{Av}(\Omega[G])\right\}
\end{gathered}
$$

\subsection{Stochastic Stability Bounds, vanishing of $\Delta_{1} G$}

We follow the method of stochastic stability as developed in [CGi].

Lemma 4.1 For every bounded observable $G$, see definition (10), we have that for every interval $\left[\beta_{1}, \beta_{2}\right]$ in the thermodynamic limit

$$
\int_{\beta_{1}}^{\beta_{2}} \Delta_{1} G d \beta=0
$$

Proof. We observe that deriving $\langle G>$ with respect to the temperature

$$
\left.-\frac{\partial<G>}{\partial \beta}=|\Lambda| \sum_{l=1}^{R}\left\{\operatorname{Av}\left(\Omega\left[h\left(\sigma^{(l)}\right) G\right]-\Omega\left[h\left(\sigma^{(l)}\right)\right] \Omega[G]\right)\right)\right\}
$$

Integrating in $d \beta$ we obtain thanks to (4.26)

$$
\int_{\beta_{1}}^{\beta_{2}} \Delta_{1} G d \beta=\frac{<G>\left(\beta_{2}\right)-<G>\left(\beta_{1}\right)}{|\Lambda|}
$$

Remembering the assumption on boundedness of function $G$ this proves the lemma.

Remark 5 The previous lemma is related to a general property of disordered systems which is known as stochastic stability (see [AC, CGi]). It says that the equilibrium state in a spin glass model is invariant under a suitable class of perturbation in all temperature intervals of continuity. 
Lemma 4.2 The following expression holds:

$$
\Delta_{1} G=-\beta<G\left[\sum_{\substack{k, l=1 \\ k \neq l}}^{R} q_{l, k}-2 R \sum_{l=1}^{R} q_{l, R+1}+R(R+1) q_{R+1, R+2}\right]>.
$$

\section{Proof.}

For each replica $l(1 \leq l \leq R)$, we evaluate separately the two terms in the right side of Eq. (4.26) by using the integration by parts (generalized Wick formula) for correlated Gaussian random variables, $x_{1}, x_{2}, \ldots, x_{n}$

$$
\operatorname{Av}\left(x_{i} \psi\left(x_{1}, \ldots, x_{n}\right)\right)=\sum_{j=1}^{n} \operatorname{Av}\left(x_{i} x_{j}\right) \operatorname{Av}\left(\frac{\partial \psi\left(x_{1}, \ldots, x_{n}\right)}{\partial x_{j}}\right) .
$$

It is convenient to denote by $p(R)$ the Gibbs-Boltzmann weight of $\mathrm{R}$ copies of the deformed system

$$
p(R)=\frac{e^{-\beta\left[\sum_{k=1}^{R} H_{\Lambda}\left(\sigma^{(k)}\right)\right]}}{[\mathcal{Z}(\beta)]^{R}},
$$

so that we have

$$
-\frac{1}{\beta} \frac{d p(R)}{d H_{\Lambda}(\tau)}=p(R)\left(\sum_{k=1}^{R} \delta_{\sigma^{(k)}, \tau}\right)-R p(R) \frac{e^{-\beta\left[H_{\Lambda}(\tau)\right]}}{[\mathcal{Z}(\beta)]} .
$$

We obtain

$$
\begin{aligned}
\operatorname{Av}\left(\Omega\left(h\left(\sigma^{(l)}\right) G\right)\right) & =\frac{1}{|\Lambda|} \operatorname{Av}\left(\sum_{\sigma^{(1)}, \ldots, \sigma^{(r)}} G H_{\Lambda}\left(\sigma^{(l)}\right) p(R)\right) \\
& =\operatorname{Av}\left(\sum_{\sigma^{(1)}, \ldots, \sigma^{(r)}} \sum_{\tau} G c_{\Lambda}\left(\sigma^{(l)}, \tau\right) \frac{d p(R)}{d H_{\Lambda}(\tau)}\right) \\
& =-\beta\left[\sum_{k=1}^{R}<G q_{l, k}>-R<G q_{l, R+1}>\right]
\end{aligned}
$$

where in (4.36) we made use of the integration by parts formula and (4.37) is obtained by (4.34). Analogously, the other term reads

$$
\operatorname{Av}\left(\Omega\left(h\left(\sigma^{(l)}\right)\right) \Omega(G)\right)=\frac{1}{|\Lambda|} \operatorname{Av}\left(\sum_{\sigma^{(l)}} \sum_{\tau^{(1)}, \ldots, \tau^{(R)}} G H_{\Lambda}\left(\sigma^{(l)}\right) p(R+1)\right)
$$




$$
\begin{aligned}
& =\operatorname{Av}\left(\sum_{\sigma^{(l)}} \sum_{\tau^{(1)}, \ldots, \tau^{(R)}} \sum_{\gamma} G c_{\Lambda}\left(\sigma^{(l)}, \gamma\right) \frac{d p(R+1)}{d H_{\Lambda}(\gamma)}\right) \\
& =-\beta\left[\sum_{k=1}^{R+1}<G q_{k, R+1}>-(R+1)<G q_{R+1, R+2}>\right]
\end{aligned}
$$

Inserting the (4.37) and (4.40) in Eq. (4.26) we finally obtain the expression (4.31).

\subsection{Martingale Bounds, vanishing of $\Delta_{2} G$}

The method of the martingale differences to prove the self averaging of the free energy, or in general to bound the fluctuations of extensive quantity, has been applied in the context of spin glasses in $[\mathrm{PS}]$ for the SK case and in [WA] in the case of finite dimensional models. Our formulation applies to both cases and extends the previous results. For instance our method includes the non summable interactions in finite dimensions [KS] and the p-spin mean field model as well as the REM [D] and GREM [DG] models.

Lemma 4.3 The free energy is a self averaging quantity, i.e. it exist a positive function $c(\beta)$ such that

$$
V(\mathcal{A})=\operatorname{Av}\left(\mathcal{A}^{2}\right)-\operatorname{Av}(\mathcal{A})^{2} \leq c(\beta)|\Lambda|
$$

Proof. For an assigned volume $\Lambda$ we enumerate by the index $k$ the interacting subsets $X$ from 1 to $N_{\Lambda}$ and considering the random partition function (2.13) we define

$$
A_{k}=\mathrm{Av}_{\leq k} \ln \mathcal{Z}(\beta),
$$

where the symbol $\mathrm{Av}_{\leq k}$ denotes the Gaussian integration performed only on the first $k$ random variables $J_{X}$. Clearly $A_{0}=\mathcal{A}(\beta)$ and $A_{N_{\Lambda}}=A(\beta)$. Introducing the quantity

$$
\Psi_{k}=A_{k}-A_{k+1},
$$

it holds

$$
\mathcal{A}-\operatorname{Av}(\mathcal{A})=\sum_{k=0}^{N_{\Lambda}-1} \Psi_{k}
$$


and

$$
V(\mathcal{A})=\sum_{k} \operatorname{Av}\left(\Psi_{k}^{2}\right)+2 \sum_{k>k^{\prime}} \operatorname{Av}\left(\Psi_{k} \Psi_{k^{\prime}}\right) .
$$

First we observe that the second sum is zero, being zero each of its terms. In fact

$$
\operatorname{Av}\left(\Psi_{k} \Psi_{k^{\prime}}\right)=\operatorname{Av}\left(\operatorname{Av}_{\leq k}\left(\Psi_{k} \Psi_{k^{\prime}}\right)\right)=\operatorname{Av}\left(\Psi_{k} \operatorname{Av}_{\leq k}\left(\Psi_{k^{\prime}}\right)\right)
$$

and

$$
\operatorname{Av}_{\leq k}\left(\Psi_{k^{\prime}}\right)=\operatorname{Av}_{\leq k}\left(A_{k^{\prime}}-A_{k^{\prime}+1}\right)=0
$$

thanks to the property

$$
\operatorname{Av}_{\leq k}\left(A_{k^{\prime}}\right)=A_{k} \quad \forall \quad k \geq k^{\prime} .
$$

We introduce now the interpolated Hamiltonian

$$
H_{\Lambda}^{(t)}(\sigma)=-\sum_{l=1}^{N_{\Lambda}} J_{l} t_{l} \sigma_{l}
$$

with

$$
t_{l}= \begin{cases}t, & \text { if } l=k+1 \\ 1, & \text { otherwise }\end{cases}
$$

and define the quantity

$$
A_{k}(t)=\mathrm{Av}_{\leq k} \ln \sum_{\sigma \in \Sigma_{\Lambda}} e^{-\beta H_{\Lambda}^{(t)}(\sigma)} .
$$

By the fundamental theorem of calculus

$$
A_{k}=A_{k}(0)+B_{k}
$$

with

$$
B_{k}=\int_{0}^{1} \frac{d A_{k}(t)}{d t} d t=\beta \int_{0}^{1} \operatorname{Av}_{\leq k} \omega_{t}\left(J_{k+1} \sigma_{k+1}\right) .
$$

We observe

$$
\begin{array}{r}
\operatorname{Av}\left(\Psi_{k}^{2}\right)=\operatorname{Av}\left(\left[A_{k}-A_{k+1}\right]^{2}\right)=\operatorname{Av}\left(\operatorname{Av}_{k+1}\left[A_{k}-\operatorname{Av}_{k+1} A_{k}\right]^{2}\right) \\
=\operatorname{Av}\left(\operatorname{Av}_{k+1}\left(A_{k}^{2}\right)-\left[\operatorname{Av}_{k+1}\left(A_{k}\right)\right]^{2}\right)
\end{array}
$$


Since $A_{k}$ and $B_{k}$ differ by a constant with respect to $A_{v_{k+1}}$ (integration with respect to the $(k+1)$-th Gaussian) we have that their variance is the same:

$$
\operatorname{Av}\left(\Psi_{k}^{2}\right)=\operatorname{Av}\left(\operatorname{Av}_{k+1}\left(A_{k}^{2}\right)-\left[\operatorname{Av}_{k+1}\left(A_{k}\right)\right]^{2}\right)=\operatorname{Av}\left(\operatorname{Av}_{k+1}\left(B_{k}^{2}\right)-\left[\operatorname{Av}_{k+1}\left(B_{k}\right)\right]^{2}\right)
$$

We will estimate separately the two terms $\operatorname{Av}\left(\operatorname{Av}_{k+1}\left(B_{k}^{2}\right)\right)$ and $\operatorname{Av}\left(\left[\operatorname{Av}_{k+1}\left(B_{k}\right)\right]^{2}\right)$. By a simple integration by parts $(4.32)$ on $J_{k+1}$ we obtain

$$
\operatorname{Av}_{k+1}\left(B_{k}\right)=\beta^{2} \Delta_{k+1}^{2} \int_{0}^{1} \operatorname{Av}_{\leq k+1}\left[1-\omega_{t}^{2}\left(\sigma_{k+1}\right)\right] t d t \leq \frac{1}{2} \beta^{2} \Delta_{k+1}^{2}
$$

which implies

$$
0 \leq \operatorname{Av}\left(\left[\operatorname{Av}_{k+1}\left(B_{k}\right)\right]^{2}\right) \leq \frac{1}{4} \beta^{4} \Delta_{k+1}^{4}
$$

Analogously we have

$$
\operatorname{Av}_{k+1}\left(B_{k}^{2}\right)=\operatorname{Av}_{k+1} \int_{0}^{1} \int_{0}^{1} \operatorname{Av}_{\leq k}\left(\omega_{t}\left(J_{k+1} \sigma_{k+1}\right)\right) \operatorname{Av}_{\leq k}\left(\omega_{s}\left(J_{k+1} \sigma_{k+1}\right)\right) s t d s d t
$$

Applying twice the integration by parts (4.32) we get

$$
\begin{aligned}
\operatorname{Av}_{k+1}\left(B_{k}^{2}\right) & =\beta^{2} \Delta_{k+1}^{2} \operatorname{Av}_{k+1} \int_{0}^{1} \int_{0}^{1} \operatorname{Av}_{\leq k}\left(\omega_{t}\left(\sigma_{k+1}\right)\right) \operatorname{Av}_{\leq k}\left(\omega_{s}\left(\sigma_{k+1}\right)\right) s t d s d t+ \\
& -2 \beta^{4} \Delta_{k+1}^{4} \operatorname{Av}_{k+1} \int_{0}^{1} \int_{0}^{1} \operatorname{Av}_{\leq k}\left(\omega_{t}\left(\sigma_{k+1}\right)\left[1-\omega_{t}^{2}\left(\sigma_{k+1}\right)\right]\right) \operatorname{Av}_{\leq k}\left(\omega_{s}\left(\sigma_{k+1}\right)\right) s t^{3} d s d t+ \\
& -2 \beta^{4} \Delta_{k+1}^{4} \operatorname{Av}_{k+1} \int_{0}^{1} \int_{0}^{1} \operatorname{Av}_{\leq k}\left(\omega_{s}\left(\sigma_{k+1}\right)\left[1-\omega_{s}^{2}\left(\sigma_{k+1}\right)\right]\right) \operatorname{Av}_{\leq k}\left(\omega_{t}\left(\sigma_{k+1}\right)\right) s^{3} t d s d t+ \\
& +2 \beta^{4} \Delta_{k+1}^{4} \operatorname{Av}_{k+1} \int_{0}^{1} \int_{0}^{1} \operatorname{Av}_{\leq k}\left[1-\omega_{s}^{2}\left(\sigma_{k+1}\right)\right] \operatorname{Av}_{\leq k}\left[1-\omega_{s}^{2}\left(\sigma_{k+1}\right)\right] s^{2} t^{2} d s d t \\
& \leq \frac{1}{4} \beta^{2} \Delta_{k+1}^{2}+\frac{13}{18} \beta^{4} \Delta_{k+1}^{4}
\end{aligned}
$$

Putting together the (4.57) and the (4.59) we find

$$
\begin{gathered}
\operatorname{Av}\left(\Psi_{k}^{2}\right) \leq \frac{1}{4} \beta^{2} \Delta_{k+1}^{2}+\frac{35}{36} \beta^{4} \Delta_{k+1}^{4} \\
V(\mathcal{A})=\sum_{k} \operatorname{Av}\left(\Psi_{k}^{2}\right) \leq \sum_{X \subset \Lambda} \frac{1}{4} \beta^{2} \Delta_{X}^{2}+\frac{35}{36} \beta^{4} \Delta_{X}^{4} .
\end{gathered}
$$

By the assumption of thermodynamic stability with the formulation (2.10) and using the inequality $\sum_{\widetilde{X}} \Delta_{\widetilde{X}}^{4} \leq\left(\sum_{\widetilde{X}} \Delta_{\widetilde{X}}^{2}\right)^{2}$ we obtain

$$
V(\mathcal{A}) \leq|\Lambda|\left(\frac{1}{4} \beta^{2} \bar{c}+\frac{35}{36} \beta^{4} \bar{c}^{2}\right)
$$


which fulfills (4.41) with $c(\beta)=\frac{1}{4} \beta^{2} \bar{c}+\frac{35}{36} \beta^{4} \bar{c}^{2}$.

Lemma 4.4 The internal energy is self averaging almost everywhere $\beta$, i.e. defining $u=\mathcal{U} /|\Lambda|$ and $V(u)=\operatorname{Av}\left(u^{2}\right)-\operatorname{Av}(u)^{2}$ it holds in the thermodynamic limit

$$
\int_{\beta_{1}}^{\beta_{2}} V(u) d \beta \rightarrow 0
$$

\section{Proof.}

The result is obtained in two steps which use general theorems of measure theory. First from lemma 4.3 we obtain the convergence to zero almost everywhere (in $\beta$ ) of the variance of the internal energy, then thanks to a bound on the variance of the internal energy we apply the Lebesgue dominated convergence theorem which gives the lemma statement. The sequence of convex functions $\mathcal{A}(\beta) /|\Lambda|$ converges a.e. (in $J$ ) to the limiting value $a(\beta)$ of its average [GT, CG] and the convergence is self averaging in the sense of lemma 4.3. By general convexity arguments $[\mathrm{RU}]$ it follows that the sequence of the derivatives $\mathcal{A}^{\prime}(\beta) /|\Lambda|$ converges to $u(\beta)=a^{\prime}(\beta)$ almost everywhere in $\beta$ and also that the convergence is self averaging. In fact the vanishing of the variance of a sequence of convex functions is inherited, in all points in which the derivative exists (which is almost everywhere for a convex function), to the sequence of its derivatives (see [S, OTW]). From lemma 4.3 we have then

$$
V(u) \rightarrow 0 \quad \beta-\text { a.e. }
$$

In order to obtain the convergence in $\beta$-average we use the Lebesgue dominated convergence theorem. In fact we prove that the sequence of variances of $u$ is uniformly bounded (in every interval $\left[\beta_{1}, \beta_{2}\right]$ ) by an integrable function of $\beta$. A lengthy but simple computation which uses again integration by parts gives

$$
\begin{aligned}
\operatorname{Av}(\mathcal{U})= & \operatorname{Av}\left(\sum_{X \subset \Lambda} J_{X} \omega\left(\sigma_{X}\right)\right)=\sum_{X \subset \Lambda} \beta \Delta_{X}^{2}\left[1-\operatorname{Av}\left(\omega^{2}\left(\sigma_{X}\right)\right)\right] \leq \beta|\Lambda| \bar{c} \\
\operatorname{Av}\left(\mathcal{U}^{2}\right) & =\operatorname{Av}\left(\sum_{X, Y \subset \Lambda} J_{X} J_{Y} \omega\left(\sigma_{X}\right) \omega\left(\sigma_{Y}\right)\right)= \\
& =\sum_{X, Y \subset \Lambda} \beta^{2} \Delta_{X}^{2} \Delta_{Y}^{2} \operatorname{Av}\left[1-\omega^{2}\left(\sigma_{X}\right)-\omega^{2}\left(\sigma_{Y}\right)+6 \omega^{2}\left(\sigma_{X}\right) \omega^{2}\left(\sigma_{Y}\right)+\right. \\
& \left.-6 \omega\left(\sigma_{X}\right) \omega\left(\sigma_{Y}\right) \omega\left(\sigma_{X} \sigma_{Y}\right)+\omega^{2}\left(\sigma_{X} \sigma_{Y}\right)\right] \leq 14 \beta^{2}|\Lambda|^{2} \bar{c}^{2}
\end{aligned}
$$


from which

$$
V(u) \leq 15 \beta^{2} \bar{c}^{2} .
$$

From this follows (4.63).

Lemma 4.5 For every bounded observable $G$, see definition (10), we have that for every interval $\left[\beta_{1}, \beta_{2}\right]$ in the thermodynamic limit

$$
\int_{\beta_{1}}^{\beta_{2}} \Delta_{2} G d \beta=0
$$

\section{Proof.}

Thanks to the Schwartz inequality

$$
\begin{gathered}
\Delta_{2} G=\operatorname{Av}(u G-\operatorname{Av}(u) \operatorname{Av}(G))=\operatorname{Av}([u-\operatorname{Av}(u)][G-\operatorname{Av}(G)])= \\
\leq \sqrt{\operatorname{Av}\left([u-\operatorname{Av}(u)]^{2}\right)} \sqrt{\operatorname{Av}\left([G-\operatorname{Av}(G)]^{2}\right)} \leq \sqrt{2} \sqrt{V(u)} \\
\left(\Delta_{2} G\right)^{2} \leq 2 V(u) \\
\left|\int_{\beta_{1}}^{\beta_{2}} \Delta_{2} G d \beta\right| \leq \sqrt{\int_{\beta_{1}}^{\beta_{2}}\left(\Delta_{2} G\right)^{2} d \beta} \sqrt{\beta_{2}-\beta_{1}} \leq \sqrt{2\left(\beta_{2}-\beta_{1}\right)} \sqrt{\int_{\beta_{1}}^{\beta_{2}} V(u) d \beta} \rightarrow 0
\end{gathered}
$$

Lemma 4.6 The following expression holds:

$$
\Delta_{2} G=-\beta R\left[\sum_{k=1}^{R}<G q_{l, R+1}>-(R+1)<G q_{R+1, R+2}>+<G><q_{1,2}>\right] .
$$

Proof. In order to obtain the $\Delta_{2} G$ we are left with the explicit evaluation of the other term in (4.27) which simply gives

$$
\begin{aligned}
\operatorname{Av}\left(\Omega\left(h\left(\sigma^{(l)}\right)\right)\right) \operatorname{Av}(\Omega(G)) & =\frac{1}{|\Lambda|} \operatorname{Av}\left(\sum_{\sigma^{(l)}} H_{\Lambda}\left(\sigma^{(l)}\right) p_{\Lambda}(1)\right)<G> \\
& =\operatorname{Av}\left(\sum_{\sigma^{(l)}} \sum_{\gamma} c_{\Lambda}\left(\sigma^{(l)}, \gamma\right) \frac{d p_{\Lambda}(1)}{d H_{\Lambda}(\gamma)}\right)<G> \\
& =-\beta<G>\left[<q_{1,1}>-<q_{1,2}>\right]
\end{aligned}
$$


Inserting the (4.40) and (4.74) in Eq. (4.27) we obtain the (4.73).

Acknowledgments. We thank S. Graffi and F. Guerra for many interesting discussions on the subject and for their suggestions. We also thank A.Bovier, C.Newman, D.Stein, M.Talagrand and F.L. Toninelli.

\section{References}

[AC] M.Aizenman, P.Contucci, "On the Stability of the Quenched state in Mean Field Spin Glass Models", J. Stat. Phys., Vol. 92, N. 5/6, 765-783, (1998).

[AiSS] M. Aizenman, R. Sims, S.L. Starr, "Extended variational principle for the Sherrington-Kirkpatrick spin-glass model", Phys. Rev. B Vol. 68, 214403 (2003)

[BCK] A.Bianchi, P.Contucci, A.Knauf, "Stochastically Stable Quenched Measures", Journ. Stat. Phys. Vol. 117, Nos. 5/6, 831-844, (2004)

[B] A. Bovier, Statistical mechanics of disordered systems, MaPhySto Lecture Notes Vol. 10 (2001), Aarhus.

[C] P.Contucci, "Toward a classification theorem for stochastically stable measures", Markov Proc. and Rel. Fields., Vol 9, N. 2, 167-176, (2002).

[C2] P.Contucci, "Replica Equivalence in the Edwards-Anderson Model", J. Phys. A: Math. Gen., Vol. 36, 10961-10966, (2003).

[CG] P. Contucci, S. Graffi, "Monotonicity and Thermodynamic Limit for Short Range Disordered Models" Journ. Stat. Phys., Vol. 115, Nos. 1/2, 581-589, (2004)

[CG2] P. Contucci, S. Graffi, "Convex Replica Symmetry Breaking from Positivity and Thermodynamic Limit", Int. Jou. Mod. Phys. B, Vol. 18, Nos. 4-5, 585-591 $(2004)$ 
[CGi] P. Contucci, C. Giardinà, "Spin-Glass Stochastic Stability: a Rigorous Proof" Annales Henri Poincare (2005), to appear

[CGi2] P. Contucci, C. Giardinà, "Factorization Properties in the 3D EdwardsAnderson Model", cond-mat/0503155

[CGI] P. Contucci, S. Graffi, S. Isola, "Mean field behaviour of spin systems with orthogonal interaction matrix", Journ. Stat. Phys., Vol. 106, N. 5/6, 895-914 $(2002)$

[CDGG] P.Contucci, M.Degli Esposti, C.Giardinà and S.Graffi, "Thermodynamical Limit for Correlated Gaussian Random Energy Models", Commun. Math. Phys., Vol. 236, 55-63, (2003).

[D] B. Derrida, "Random energy model: limit of a family of disordered system", Phys. Rev. Lett. 4579 (1980)

[DG] B.Derrida, E.Gardner, "Solution of the generalized random energy model" J. Phys. C 19 2253-2274 (1986)

[EA] S.Edwards and P.W.Anderson "Theory of spin glasses", J. Phys. F, Vol. 5, 965974, (1975)

[FMPP1] S.Franz, M.Mezard, G.Parisi, L.Peliti, "Measuring equilibrium properties in aging systems", Phys. Rev. Lett., Vol. 81, 1758 (1998).

[FMPP2] S.Franz, M.Mezard, G.Parisi, L.Peliti, "The response of glassy systems to random perturbations: A bridge between equilibrium and off-equilibrium", J. Stat. Phys. Vol. 97, N. 3/4, 459-488 (1999).

[G1] F. Guerra, "Broken Replica Symmetry Bounds in the Mean Field Spin Glass Model", Comm. Math. Phys., Vol. 233, 1 - 12, (2003)

[G2] F.Guerra, "About the overlap distribution in a mean field spin glass model", Int. J. Phys. B, Vol. 10, 1675-1684 (1997). 
[GG] S. Ghirlanda, F. Guerra, "General properties of overlap probability distributions in disordered spin systems. Towards Parisi ultrametricity", J. Phys. A: Math. Gen., Vol. 31, 9149-9155 (1998).

[GT] F. Guerra, F.L. Toninelli, "The Thermodynamic Limit in Mean Field Spin Glass Models", Comm. Math. Phys., Vol. 230, 71-79, (2002)

[KS] K.M Khanin, Ya.G. Sinai, "Existence of free energy for models with long-range random Hamiltonians", Journ. Stat. Phys., Vol. 20, 573-584, (1979)

[MPV] M.Mezard, G.Parisi, M.A.Virasoro, Spin Glass theory and beyond, World Scientific, Singapore (1987).

[MPRRZ] E. Marinari, G. Parisi, F. Ricci-Tersenghi, J. Ruiz-Lorenzo and F. Zuliani, Jour. Stat. Phys. 98973 (2000)

[NS] C.M. Newman, D.L. Stein, "Local vs. Global Variables for Spin Glasses", condmat/0503344

[OTW] E. Orlandini, M.C. Tesi, S.G. Whittington, "Self averaging in the statistical mechanics of some lattice models", J. Phys. A: Math. Gen. Vol. 35 1-9 (2002)

[Pa] G.Parisi, "On the probabilistic formulation of the replica approach to spin glasses", Int. Jou. Mod. Phys. B, Vol. 18, 733-744, (2004).

[PS] L.A. Pastur, M.V. Scherbina, "Absence of self-averaging of the order parameter in the Sherrington-Kirkpatrick model", Jour. Stat. Phys. Vol 62, Nos 1/2, 1-19, (1991)

[RU] D. Ruelle, Statistical Mechanics, Rigorous Results, W.A. Benjamin, New York 1969

[S] M. Scherbina, "On the replica symmetric solution for the SherringtonKirkpatrick model", Helv. Phys. Acta Vol. 70, 838-853 (1997) 
[SK] D.Sherrington and S.Kirkpatrick, "Solvable model of a spin-glass," Phys. Rev. Lett., Vol. 35, 1792-1796 (1975).

[T1] M. Talagrand, "The Parisi formula", Annals of Mathematics (2005), to appear.

[T2] M.Talagrand, Spin glasses: a challenge for mathematicians, Berlin, Springer (2003).

[WA] J. Wehr, M. Aizenman, "Fluctuations of extensive functions of quenched random couplings", Journ. Stat. Phys. Vol. 60 Nos. 3/4, 287-305 (1990) 